\title{
The impact of minimally invasive restorative techniques on perception of dental pain among pregnant women: a randomized controlled clinical trial
}

\author{
May M. Adham", Mona K. El Kashlan, Wafaa E. Abdelaziz ${ }^{1}$ and Ahmed S. Rashad²
}

\begin{abstract}
Background: The public dental care sector is striving to fulfill the preventive and restorative needs of Egyptians, including pregnant women, who may not receive timely care due to misconceptions about dental treatment during pregnancy. Because of this, they are likely to suffer dental pain, with higher risk of infection affecting their offsprings.

Aim of the study: To compare the effectiveness of chemo-mechanical caries removal using Papacarie-Duo and Atraumatic Restorative Treatment (ART) in reducing dental pain among pregnant women.

Materials and methods: A randomized controlled clinical trial was conducted, in 2019, and included 162 pregnant women visiting family health centers in Alexandria, Egypt, with dental pain due to dental caries not extending to pulp. Patients were randomly assigned to Papacarie-Duo group $(n=82)$ and ART group $(n=80)$ after stratification by number of treated surfaces. The outcome variables were reduction in pain assessed using Visual Analogue Scale (VAS), satisfaction with treatment, and time taken for dental caries removal. T test/ Mann Whitney $\mathrm{U}$ test were used to compare groups and Freidman test was used to compare change across time.

Results: Pain reduction was significantly greater in the Papacarie-Duo than the ART group ( $81.55 \%$ and $69.43 \%$, $P=0.001$ ). Patients in the Papacarie-Duo group were significantly more satisfied with treatment than those in the ART, immediately after treatment (mean $=9.60$ and 8.00, $P=<0.01$ ) and after 6 months (mean $=9.63$ and 8.16, $P=<0.01$ ). Significantly less excavation time was recorded in the Papacarie-Duo group than in the ART group (mean $=10.38$ and $11.56 \min , P=<0.01)$.
\end{abstract}

Conclusion: Chemo-mechanical caries removal using Papacarie-Duo is more effective in reducing dental pain, in pregnant women, and is associated with more satisfaction and less excavation time than ART.

Trial registration: ID NCT04573608 (https://clinicaltrials.gov/); 5/10/2020, retrospective registration.

Keywords: Chemo-mechanical caries removal, Papacarie-duo, Pregnant women, Atraumatic restorative treatment, Minimal invasive dentistry, Dental pain, Patient satisfaction, Excavation time

*Correspondence: may.adham00@gmail.com

1 Department of Pediatric Dentistry and Dental Public Health,

Faculty of Dentistry, Alexandria University, Champolion St., Azarita,

Alexandria 21527, Egypt

Full list of author information is available at the end of the article

\section{Background}

Physical and hormonal changes affect pregnant women in many ways and the oral cavity is no exception. There are many common oral problems during this stage such as pregnancy gingivitis, benign gingival lesions, tooth mobility, tooth erosion, periodontitis and dental caries 
[1]. Dental caries is a biofilm-mediated, diet modulated, multifactorial, non-communicable, dynamic disease resulting in net mineral loss of dental hard tissues. It is determined by biological, behavioral, psychosocial, and environmental factors. As a consequence of this process, a caries lesion develops [2, 3]. The increased risk for developing dental caries during this stage can occur due to several factors; the oral cavity is characterized by an acidic environment due to an inflammatory response brought about by vomiting, gastric-reflux and changes in salivary composition. This is further complicated by the high sugar cravings that are common during this period as well as the limited attention to oral health [4]. Thus, the presence of untreated dental caries reflects unmet treatment need rather than higher incidence of disease during this period. Studies showed that if dental caries is left untreated, may in some cases result in further inflammatory complications which could influence pregnancy outcomes like preterm birth and/or low birth weight [5-7].

Despite these consequences, pregnant women, sometimes, do not seek dental treatment, not only due to misconceptions about the safety of dental procedures during pregnancy but also because some dentists may be reluctant to provide dental treatment [8].

In Rio de Janeiro, Brazil, 39\% of the participating pregnant women experienced dental pain, which affected their daily normal activities [9]. Whereas, in South Brazil, prevalence of dental pain was found to be $54.9 \%$ [10]. Unlike other types of pain experienced during pregnancy, pain due to dental caries can be avoided by early treatment instead of analgesics that may affect the infant's health [11].

There has been an increased interest in the use of minimally invasive restorative procedures as an alternative to traditional rotary techniques of dental caries removal to overcome its various disadvantages such as vibration, pressure and heat generation. These minimally invasive methods include chemo-mechanical agents and atraumatic restorative treatment (ART) [12].

The ART technique involves dental caries removal using hand instruments, followed by cavity filling using glass ionomer. There is no need for anesthesia and pain is reduced to minimum. This approach was originally developed for use outside the dental clinic, in remote areas with no electricity $[13,14]$. It can also be used for children, elderly population, patients with special needs and those who experience dental anxiety [15]. Shenoy et al. [16] assessed pregnant women's response to ART, in primary health care centers, and reported a high level of satisfaction with the procedure where none of the participants felt pain during treatment.
Chemo-mechanical caries removal, on the other hand, involves the use of chemical solutions which soften dental caries and facilitate its removal using hand instruments. There are two categories of chemo-mechanical caries removal agents: sodium hypochlorite $(\mathrm{NaOCl})$ such as Carisolv, and enzyme-based agents where Papacarie is considered its most common agent [17]. Papacarie-Duo, the newer version of the product, was developed in 2011, and has greater viscosity allowing more precise application, longer shelf life with no need to be refrigerated.

Most studies involving oral health of pregnant women focused on periodontal conditions [18-21], while few studies were concerned with pain caused by dental caries $[10,16]$. Furthermore, several studies compared conventional drilling and chemo-mechanical treatments [22-25]. However, only few studies compared ART and chemo-mechanical treatment, and those were mostly conducted in children. Only one pilot study compared chemo-mechanical treatment using Carisolv and ART, among 50 pregnant women, in order to assess the longevity of glass ionomer restoration [26]. Given the advantage of Papacarie-Duo over Carisolv in relation to lower cost and time taken in dental caries excavation [17], it may have added advantage in countries with limited resources and facilities, like Egypt. Therefore, if its effectiveness is proven, Papacarie-Duo can be a feasible method to treat dental caries and reduce dental pain among pregnant women during routine prenatal care in family health centers.

The aim of the study was, thus, to compare the effectiveness of two minimally invasive caries removal techniques: chemo-mechanical (Papacarie-Duo) and ART, in pregnant women, regarding pain reduction after one month and six months, their satisfaction with the treatment procedure and time taken for dental caries removal. The null hypothesis was that there would be no statistically significant difference between the two modalities in pain reduction.

\section{Methods \\ Study design}

A randomized, two parallel arms, controlled clinical trial was conducted among pregnant women attending family health centers for their routine prenatal care in Alexandria, Egypt, from January to October 2019.

The study was conducted in accordance with the CONSORT guidelines[27] and Helsinki declaration; and it was approved by the Research Ethics Committee, Faculty of Dentistry, Alexandria University (IRB 00010556-IORG 0008839). Permission to access the family health facilities was obtained from the Health Directorate of Alexandria. Signed written informed consents were obtained from patients after explaining the aim of the study, risks, 
benefits and confirming confidentiality of their response as well as their right to withdraw at any time. The trial was registered at clinicaltrial.gov (NCT04573608) on $5 / 10 / 2020$.

\section{Participants}

Pregnant women were eligible to participate if they were in the first or second trimester, had at least mild dental pain as identified by a score of at least $5 \mathrm{~mm}$ on a Visual Analogue Scale (VAS) 100-mm-long [28], and with at least one carious lesion involving dentine clinically classified as a shallow or medium cavity. This cavity should be accessible to hand instruments (International Caries Detection and Assessment System (ICDAS) score $=5$ or 6) [29]). Pregnant women with acute pulpitis, swelling or fistula as well as uncooperative patients, those having severe gingivitis (Gingival Index (GI) score $=3$ [30]), those who are unable to read and/or write and those who refused to participate were excluded from the study.

Sample size was based on assuming a 5\% alpha error, $20 \%$ beta error and reported percentage of patients with pain after using chemo-mechanical caries removal $=68 \%$ and $35 \%$ after ART [31]. The minimum required number of patients was estimated to be 33 per group [32]. This was increased to 40 to make up for loss to follow up. Pain and effect of the two treatment modalities was assumed to be affected by the extent of dental caries and; hence, stratified by the number of surfaces affected by dental caries into single and multi-surface lesions, creating two strata per group. The number of participants, therefore, per group was 80 with a total of 160 participants.

\section{Randomization}

A computer-generated list of random numbers was used to randomly assign patients into one of the two study groups in a ratio 1:1 in blocks of four [33]. The allocation sequence was concealed from the researcher administering the intervention in sequentially numbered, opaque, sealed envelopes [34]. Blinding of participants was not possible due to the difference between the two techniques.

\section{Interventions}

\section{Group I: Papacarie-Duo}

Papacarie was introduced into the cavity using the applicator and left for $40 \mathrm{~s}$ and a blunt excavator was used to remove the softened dentin. The remaining gel was removed using a cotton pellet. When there was no change in gel color, the cavity was considered caries free [23]. The cavity was then filled with high viscosity glass ionomer cement (GIC) in an encapsulated form (Riva Self-Cure, SDI Limited, Bayswater, VIC, Australia). A mechanical mixer was used to mix the capsule for $10 \mathrm{~s}$, the capsule was placed into the applicator to apply the GIC into the cavity. For occluso-proximal cavities, a matrix strip with a wooden wedge was used to provide the appropriate contour of the restoration. A gloved finger was used to apply pressure on the GIC for one minute and occlusion was checked and excess material was removed [35].

\section{Group II: ART}

The tooth was cleaned with a wet cotton pellet to remove debris and plaque, dental caries was then removed using sharp spoon excavators (Darby-Perry \#220/221, \#17 DE, $\mathrm{Hu}$-Friedy, Chicago, USA), followed by cleaning the cavity using a small wet cotton pellet and finally dried with a dry cotton pellet. The cavity was considered caries-free when a leather-hard texture was reached and the excavator did not stick anymore [14]. The GIC was used to restore the cavity using the same technique described for the other group [33].

\section{Outcomes assessment}

- Difference in pain was measured using Visual Analogue Scale (VAS) at baseline, after one month and a six months period. The scale is represented by a 100-mm-long horizontal line labeled "no pain" at one end and "worst pain" at the other end. Participants were asked to mark the place on the line representing their level of pain [28].

- Satisfaction with treatment was assessed using two questions: the first question was immediately following treatment: "Was the treatment carried out according to your expectations?" The second question was after 6 months" "Has the treatment solved the problem of your teeth?" Each question was answered on a 10-point scale, with lower values indicating a negative perspective and higher values indicating a positive experience [36].

- Time to remove dental caries was recorded using a stop watch from the start of excavation up to complete dental caries removal [37]

- To control for potential confounders, information about age, education level, pregnancy stage, brushing frequency and last dental visit as well as clinical oral health indicators like number of decayed teeth, gingival index and plaque index were collected using the Arabic version of the WHO oral health assessment questionnaire for adults which was previously validated [38]. Clinical examination assessed the number of decayed teeth based on WHO criteria [39]. The amount of dental plaque and the gingival condition were both assessed using Silness and Loe [40] and Loe and Silness criteria [30]. 


\section{Statistical analysis}

SPSS (Version 24.0, IBM Corp., Armonk, N.Y., USA) was used for data analysis. Quantitative variables were checked for normality using Shapiro Wilks tests, histograms and QQ plots. Descriptive statistics were displayed as frequencies and percentages for categorical variables and means and standard deviations for quantitative variables. Independent $t$ test or Mann Whitney $\mathrm{U}$ test (depending on normality) was used to assess differences in quantitative variables: age, number of decayed teeth, gingival index, plaque index, pain and satisfaction scores. Chi square was used for categorical variables: education level, brushing frequency and last dental visit. Friedman test used to assess change in VAS across time in each group. An intention-to-treat analysis was performed by assigning the worst pain and satisfaction scores to those lost to follow up using worst case scenario analysis. Significance level was set at $P<0.05$.

\section{Results}

Among 250 pregnant women assessed for eligibility, 162 participants fulfilled the inclusion criteria and were randomly allocated to Papacarie-Duo group $(\mathrm{n}=82)$ or ART group $(n=80)$ (Fig. 1). The dropout rate after six months was $14.6 \%$ and $17.5 \%$, respectively.

Table 1 presents general characteristics of the study sample, mean age (SD) of pregnant women in the Papacarie-Duo and ART groups were: 27.1 (2.82) and 26.3 (3.18). The majority of pregnant women received secondary education (58.5\% and $63.8 \%$ respectively), were in the second trimester ( $64.6 \%$ and $73.8 \%$ respectively), brushed at least once per day (53.7\% and $42.5 \%$ respectively) and slightly more pregnant women in the ART group than those in the Papacarie-Duo group visited the dentist within the last year (57.5\% $47.6 \%$ respectively).

Regarding the consumption of sugar at least once per day, tea with sugar was the most common source of sugar reported by $74.4 \%$ of women in the Papacarie-Duo group and $67.5 \%$ of those in the ART, followed by sweets/candies: (54.9\% and $60 \%$ respectively). Consumption of fresh

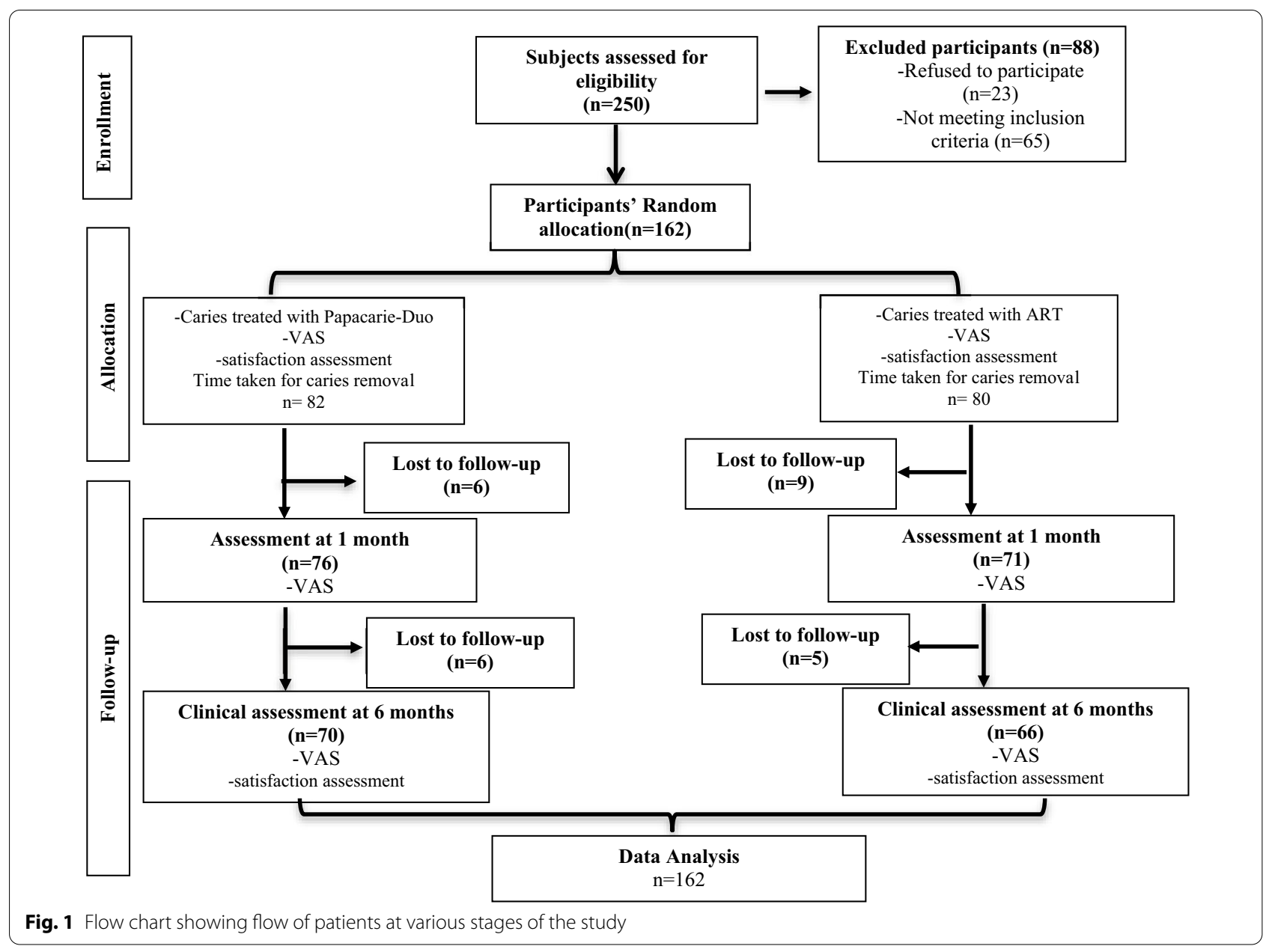


Table 1 Comparison of pregnant women in the study groups regarding personal background, oral health practices, and oral health indicators

\begin{tabular}{|c|c|c|}
\hline & $\begin{array}{l}\text { Papacarie-Duo } \\
n=82\end{array}$ & $\begin{array}{l}\text { ART } \\
n=80\end{array}$ \\
\hline Age mean (SD) & $27.1(2.82)$ & $26.3(3.18)$ \\
\hline \multicolumn{3}{|l|}{ Education n (\%) } \\
\hline Primary education & $12(14.6 \%)$ & $9(11.3 \%)$ \\
\hline Secondary education & $48(58.5 \%)$ & $51(63.8 \%)$ \\
\hline Higher education & $22(26.8 \%)$ & $20(25 \%)$ \\
\hline \multicolumn{3}{|l|}{ Brushing frequency $n(\%)$} \\
\hline Less than once per day & $38(46.3 \%)$ & $46(57.5 \%)$ \\
\hline At least once per day & $44(53.7 \%)$ & $34(42.5 \%)$ \\
\hline \multicolumn{3}{|l|}{ Last Dental visit n (\%) } \\
\hline Within the last year & $39(47.6 \%)$ & $46(57.5 \%)$ \\
\hline More than 1 year, less than 5 years & $38(46.3 \%)$ & $32(40 \%)$ \\
\hline More than 5 years & $5(6.1 \%)$ & $2(2.5 \%)$ \\
\hline \multicolumn{3}{|l|}{ Trimester n (\%) } \\
\hline First trimester n (\%) & $29(35.4 \%)$ & $21(26.3 \%)$ \\
\hline Second trimester $\mathrm{n}(\%)$ & $53(64.6 \%)$ & $59(73.8 \%)$ \\
\hline \multicolumn{3}{|l|}{ Diet } \\
\hline \multicolumn{3}{|l|}{ Sweets/candies } \\
\hline At least daily & $45(54.9 \%)$ & $48(60 \%)$ \\
\hline Less than daily & $37(45.1 \%)$ & $32(40 \%)$ \\
\hline \multicolumn{3}{|l|}{ Tea with sugar } \\
\hline At least daily & $61(74.4 \%)$ & $54(67.5 \%)$ \\
\hline Less than daily & $21(25.6 \%)$ & $26(32.5 \%)$ \\
\hline \multicolumn{3}{|l|}{ Fruits } \\
\hline At least daily & $30(36.6 \%)$ & $29(36.3 \%)$ \\
\hline Less than daily & $52(63.4 \%)$ & $51(63.8 \%)$ \\
\hline No. of decayed teeth mean (SD) & $2.91(0.86)$ & $2.71(0.92)$ \\
\hline Gingival Index mean (SD) & $1.54(0.32)$ & $1.43(0.29)$ \\
\hline Plaque Index mean (SD) & $1.69(0.34)$ & $1.65(0.29)$ \\
\hline \multicolumn{3}{|l|}{ Type of cavities n (\%) } \\
\hline One-surface cavities & $45(54.9 \%)$ & $41(51.3 \%)$ \\
\hline Multi-surfaces cavities & $37(45.1 \%)$ & $39(48.8 \%)$ \\
\hline
\end{tabular}

*Statistically significant at $P<0.05$

fruits at least once daily was less common: (36.6\% and $36.3 \%$ respectively).

There were no differences between pregnant women in the Papacarie-Duo and ART groups regarding number of decayed teeth: mean $(\mathrm{SD})=2.91(0.86)$ and $2.71(0.92)$, plaque accumulation: mean $(\mathrm{SD})=1.69(0.34)$ and 1.65 (0.29), or extent of dental caries: single surface $=54.9 \%$ and $51.3 \%$. Women in the Papacarie-Duo had more gingivitis than those in the ART group: mean $(\mathrm{SD})=1.54$ (0.32) and $1.43(0.29)$.

Table 2 shows no significance difference in pain perception between pregnant women in the Papacarie-Duo and ART groups at baseline: mean $(\mathrm{SD})=22.38(11.53)$ and
Table 2 Difference in pain scores between groups and across time

\begin{tabular}{|c|c|c|c|}
\hline & $\begin{array}{l}\text { Papacarie-Duo } \\
\text { mean (SD) }\end{array}$ & $\begin{array}{l}\text { ART } \\
\text { mean (SD) }\end{array}$ & $P$ value \\
\hline \multicolumn{4}{|c|}{ One surface cavities ( $n=86$ ) } \\
\hline Baseline & $18.33(11.28)^{\mathrm{a}}$ & $18.29(10.22)^{a}$ & 0.986 \\
\hline 1 month & $3.67(6.16)^{b}$ & $5.85(7.15)^{b}$ & 0.100 \\
\hline 6 months & $2.33(4.88)^{b}$ & $4.88(6.66)^{b}$ & $0.044^{*}$ \\
\hline$P$ value & $<0.001^{*}$ & $<0.001^{*}$ & \\
\hline Percent reduction & $89.53(23.78)$ & $79.41(29.49)$ & 0.084 \\
\hline \multicolumn{4}{|c|}{ Multi- surface cavities $(n=76)$} \\
\hline Baseline & $27.3(9.9)^{\mathrm{a}}$ & $29.49(10.5)^{\mathrm{a}}$ & 0.353 \\
\hline 1 month & $8.11(7.11)^{b}$ & $13.46(8.75)^{b}$ & $0.008^{*}$ \\
\hline 6 months & $7.43(7.32)^{b}$ & $12.82(9.23)^{b}$ & $0.012^{*}$ \\
\hline$P$ value & $<0.001^{*}$ & $<0.001^{*}$ & \\
\hline Percent reduction & $72.07(31.67)$ & $58.93(27.91)$ & $0.049^{*}$ \\
\hline \multicolumn{4}{|l|}{ All cavities $(n=162)$} \\
\hline Baseline & $22.38(11.53)^{\mathrm{a}}$ & $23.75(11.73)^{\mathrm{a}}$ & 0.454 \\
\hline 1 month & $5.67(6.93)^{b}$ & $9.56(8.79)^{b}$ & $0.003^{*}$ \\
\hline 6 months & $4.63(6.56)^{b}$ & $8.75(8.9)^{b}$ & $0.002^{*}$ \\
\hline$P$ value & $<0.001^{*}$ & $<0.001^{*}$ & \\
\hline Percent reduction & $81.55(28.84)$ & $69.43(30.35)$ & $0.01^{*}$ \\
\hline
\end{tabular}

23.75 (11.73), $P=0.454$, including those with single surface cavities: 18.33 (11.28) versus 18.29 (10.22), $P=0.987$ and those with multiple surfaces cavities: 27.3 (9.9) versus 29.49 (10.5), $P=0.353$.

At one month, women in the Papacarie-duo group had significantly less pain than women in the ART group regarding all cavities: mean $(\mathrm{SD})=5.67(6.93)$ and 9.56 (8.79), $P=0.003$ and in multiple surfaces cavities: mean $(\mathrm{SD})=8.11$ (7.11) and 13.46 (8.75), $P=0.008$. No significant difference was noted between groups among women with single surface cavities: 3.67 (6.16) and 5.85 (7.15), $P=0.100$.

After 6 months, women in the Papacarie-duo group had significantly less pain than those in the ART group whether in the whole sample: mean $(\mathrm{SD})=4.63(6.56)$ and 8.75 (8.9), $P=0.002$, in those with single surface cavities: 2.33 (4.88) and $4.88(6.66), P=0.044$ or in those with multiple surface cavities: 7.43 (7.32) and 12.82 (9.23), $P=0.012$.

The reduction in pain from baseline to one and 6 months was statistically significant in both groups $(P=<0.001)$. The difference in the percent reduction from baseline to 6 months between the groups was statistically significant for the whole sample: 81.55 (28.84) and 69.43 (30.35), $P=0.01$, for women with multiple 
surface cavities: 72.07 (31.67) and 58.93 (27.9), $P=0.04$ but not among women with single surface cavities: 89.53 (23.78) and 79.41 (29.49), $P=0.084$.

The mean (SD) satisfaction scores immediately after treatment and after 6 months were significantly higher in the Papacarie-Duo: $9.6(0.68)$ and $9.63(0.59)$ than in the ART group: $8(0.87)$ and $8.16(0.89), P<0.001$, whether among women with single surface cavities (Papacarie-Duo: $9.84(0.42)$ and $9.84(0.37)$ versus ART: $8.32(0.93)$ and $8.27(0.89), P<0.001)$ or with multiple surface cavities: Papacarie-Duo: $9.3(0.81)$ and 9.38 (0.72) versus ART: 7.67 (0.66) and 8.05 (0.89), $P<0.001$. The change in satisfaction across time in the two groups was not statistically significant for the whole sample $(P=0.320$ and 0.096 for Papacarie-Duo and ART, respectively) and for women with single surface cavities $(P=1.00$ and 0.728 , respectively). In women with multiple surfaces cavities, there was no significant difference in the Papacarie-Duo $(P=0.183)$, while in the ART group, the satisfaction after 6 months increased significantly $(P=0.004)$, Table 3 .

A significant negative moderate correlation was found between pain and satisfaction at 6 months $(\mathrm{r}=-0.339$, $P=<0.001$ ) indicating that patients who reported less pain were more satisfied with the treatment provided.

The mean (SD) time in minutes taken for dental caries removal was significantly less in the PapacarieDuo-duo: 10.38 (1.94) for all cavities, 9.36 (1.8) for one surface cavities and 11.62 (1.28) for multiple surfaces cavities than in the ART group: 11.56 (1.62) for all cavities, 10.71 (1.37) for one surface cavities and 12.46 (1.37) for multiple surfaces cavities, $P=<0.001$, Table 4.

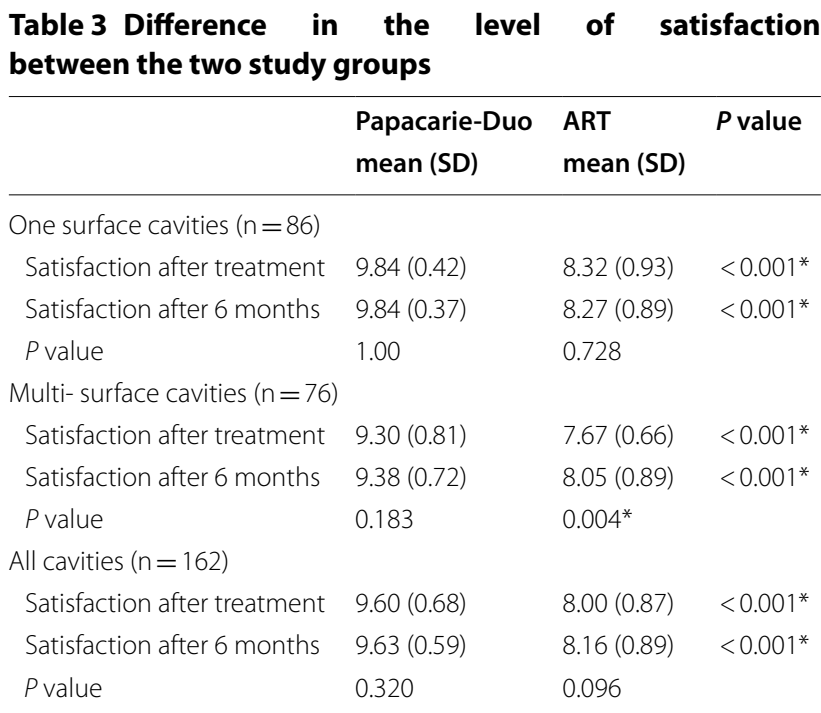

*Statistically significant at $P \leq 0.05$
Table 4 Comparison of time in minutes for dental caries removal in Papacarie-Duo and ART groups

\begin{tabular}{rllr}
\hline & $\begin{array}{l}\text { Papacarie-Duo } \\
\text { Mean (SD) }\end{array}$ & $\begin{array}{l}\text { ART } \\
\text { Mean (SD) }\end{array}$ & \\
\hline One surface $(n=86)$ & $9.36(1.8)$ & $10.71(1.37)$ & $<0.001^{*}$ \\
Multi-surfaces $(n=76)$ & $11.62(1.28)$ & $12.46(1.37)$ & $0.007^{*}$ \\
All cavities $(n=162)$ & $10.38(1.94)$ & $11.56(1.62)$ & $<0.001^{*}$ \\
\hline
\end{tabular}

*Statistically significant at $P \leq 0.05$

\section{Discussion}

Papacarie-Duo was associated with significantly greater pain reduction, higher satisfaction and less time of dental caries excavation than ART in pregnant women. The greater effectiveness of Papacarie-Duo was more observed in multi-surface cavities when pain and excavation time were considered whereas greater satisfaction compared to ART was reported after 6 months in one surface cavities. The null hypothesis of the study can, therefore, be rejected. The current findings have implications for the dental care of women who suffer from dental pain during pregnancy and are reluctant to seek conventional treatment for fear that it may harm their babies. Even if dental caries extends to more than one surface, it can still be restored with Papacarie-Duo if adequate access for the instruments can be ensured. The duration of the trial shows that this treatment can adequately last throughout the period of pregnancy after which other types of treatment can be provided if needed. Further studies with longer follow up and with economic evaluation are needed to inform policy decision makers about the use of Papacarie-Duo as an efficient restorative modality in low-resource settings.

Pain reduction was greater in multi-surfaces cavities treated with Papacarie-Duo than ART whereas there was no difference in one surface cavities. This may be attributed to the components of the gel that contains papain enzyme which has an anti-inflammatory, bactericidal and bacteriostatic action. Papacarie-Duo removes carious tissues without affecting the sound collagen fibers in the affected normal dentin, and as a result, less instrumentation is required with less pain generated during excavation [41]. This finding agrees with Ericson et al. [42] who showed that the chemo-mechanical approach is effective and more comfortable for patients compared to ART where operators sometimes tend to "dig" excavators into the hard carious dentine breaking off hard dentine pieces not only the leathery-firm dentin, thereby opening more dentine tubules which induces pain [43].

The present study is the first to evaluate the effect of two minimally invasive restorative techniques (Papacarie and ART) on pain reduction and satisfaction among adults. Only one study by Barata et al. [26] compared 
ART to chemo-mechanical caries removal using Carisolv in pregnant women, however, they assessed restoration longevity not pain and found similar clinical performance of the two methods after 12 months. Another study concluded that both Carisolv and Papacarie were clinically efficient in dental caries removal among those aged 20-40 years, while Papacarie was better regarding time taken and the volume of excavated carious tissues [41].

In the present study, there was a significant increase in satisfaction among patients with multiple surfaces cavities treated by ART, after 6 months, but not among those treated with Papacarie-Duo. This may be attributed to the fact that some patients felt relatively mild pain during excavation in larger cavities and thus longer time was taken to completely remove dental caries than in single surface cavities. This led to initially lower levels of satisfaction with subsequent potential for improved satisfaction as time passed and dental pain resolved. Although direct comparison is not possible, these findings were in line with Anegundi et al. [44] who reported that $60 \%$ of participants preferred Papacarie compared to $36.7 \%$ preferring traditional methods and Goyal et al. [45] who reported that $80 \%$ preferred Papacarie caries removal over the conventional drilling. Furthermore, the study conducted by Kumar et al. [41] on adult population comparing Papacarie and Carisolv revealed that patients, in both groups, were satisfied with the treatment provided as no pain was reported during dental caries removal. Patients' satisfaction may have been partially related to the dental pain reported at 6 months. This is supported by the significant negative correlation between pain and satisfaction, reported in the current study, at 6 months.

The present study revealed significantly shorter time of excavation in the Papacarie-duo group than in the ART, in both single and multiple surface cavities. This is because Papacarie-Duo softens dental caries so that it is easily removed. This is comparable to other studies of permanent dentition, in adults, where the time for excavation in the present study was slightly less in the Papacarie-Duo group as compared to that reported by Kumar et al. [41] and less than that recorded for Carisolv by Barata et al. [26].

One of the limitations of the study is that blinding could not be applied due to the differences between the two techniques. Also, patients who received PapacarieDuo may have over-reported their satisfaction because of their attraction to the new material and some patients had difficulty translating pain to a linear scale. There was a slight difference between groups in the severity of gingivitis. However, both groups were in the mild to moderate level of gingival inflammation and thus, this minor difference is unlikely to affect the study conclusions. Also, in the Papacarie-Duo group, the cavity was considered caries free when the gel stayed clear. On the other hand, in the ART group, excavating till hard dentin was reached might have been responsible for greater pain felt with less pain reduction reported [3, 43]. Future studies assessing these different non-invasive methods should consider having similar criteria of declaring the cavity caries free. In addition, chemomechanical caries removal can be used only if cavities are open so that an excavator can be used which limits the generalizability of findings only to those with this special form of cavities.

Papacarie-Duo is available at low cost $(73 \$$ per tube which may be used for 25 applications) providing a cost reduction of $42 \%$ compared to traditional drilling method [46]. Although no data are available about the market availability of Papacarie per country, research shows that studies about Papacarie are conducted in South America, Europe, India, Pakistan, United States and Egypt [17].

This study was performed in public healthcare centers which mainly serve the disadvantaged communities. Hence, this new low-cost line of treatment is suggested for use in remote areas, deprived from proper facilities and equipment. In addition, this study aids in filling the knowledge gap by providing evidence about the effectiveness of minimally invasive caries treatment in adults which makes possible the translation of the results of scientific research to clinical practice. Future studies, based on action research, are needed to evaluate the feasibility and cost-effectiveness of integrating minimally invasive caries removal into comprehensive dental care programs for pregnant women.

\section{Conclusion}

Chemo-mechanical caries removal using Papacarie-Duo is more effective in reducing dental pain, in pregnant women, and is associated with more satisfaction and less excavation time than ART which offers an alternative low-cost method for treatment of dental caries among women who suffer from dental pain during pregnancy but and are reluctant to seek conventional treatment.

\section{Abbreviations}

ART: Atraumatic Restorative Treatment; VAS: Visual Analogue Scale; ICDAS: International Caries Detection and Assessment System; GI: Gingival Index; GIC: Glass lonomer Cement; SPSS: Statistical Package for Social Sciences.

\section{Acknowledgements}

The authors would like to express deep gratitude and appreciation to Prof. Maha El Tantawi for her valuable and constructive suggestions during the planning and development of this research work.

\section{Authors' contributions}

MA conceptualized the study, collected data, applied the intervention, developed and implemented the statistical analysis and wrote the manuscript draft. $M E, W A$ and AR helped designing the study and contributed to the review and editing of the manuscript. All authors read and approved the final manuscript. 


\section{Funding}

The research did not receive any fund from public, commercial or not-forprofit sectors.

\section{Availability of data and materials}

The dataset used in this research is available at synapse.org under the title: The impact of minimally invasive restorative techniques on perception of dental pain among pregnant women. Synapse ID: syn23521994. User name: @may. adham.

\section{Ethics approval and consent to participate}

This study was approved by the Research Ethics Committee, Faculty of Dentistry, Alexandria University (IRB 00010556-IORG 0008839) and was registered under the protocol ID NCT04573608 (https://clinicaltrials.gov/) according to the CONSORT statement of the updated guidelines for reporting randomized clinical trials. Each participant was provided with a written informed consent form. All the information including personal details and clinical information that have been used for this study were de-identified.

\section{Consent for publication}

Not applicable.

\section{Competing interests}

Authors declare that they have no competing interests.

\section{Author details}

${ }^{1}$ Department of Pediatric Dentistry and Dental Public Health, Faculty of Dentistry, Alexandria University, Champolion St., Azarita, Alexandria 21527, Egypt. ${ }^{2}$ Department of Economics, Faculty of Commerce, Damanhour University, Damanhour, Egypt.

Received: 17 October 2020 Accepted: 1 February 2021

Published online: 18 February 2021

\section{References}

1. Gajendra S, Kumar JV. Oral health and pregnancy: a review. NY State Dent J. 2004;70:40-4.

2. Simón-Soro A, Mira A. Solving the etiology of dental caries. Trends Microbiol. 2015;23:76-82.

3. Machiulskiene V, Campus G, Carvalho JC, Dige I, Ekstrand KR, JablonskiMomeni A, et al. Terminology of dental caries and dental caries management: consensus report of a workshop organized by ORCA and cariology research group of IADR. Caries Res. 2020;54:7-14.

4. Russell SL, Mayberry LJ. Pregnancy and oral health: a review and recommendations to reduce gaps in practice and research. MCN Am J Matern Child Nurs. 2008;33:32-7.

5. Dasanayake AP, Li Y, Wiener H, Ruby JD, Lee M-J. Salivary Actinomyces naeslundii genospecies 2 and Lactobacillus casei levels predict pregnancy outcomes. J Periodontol. 2005;76:171-7.

6. Saraiva MCD, Bettiol H, Barbieri MA, Silva AA. Are intrauterine growth restriction and preterm birth associated with dental caries? Community Dent Oral Epidemiol. 2007;35:364-76.

7. Harjunmaa U, Järnstedt J, Alho L, Dewey KG, Cheung YB, Deitchler M, et al. Association between maternal dental periapical infections and pregnancy outcomes: results from a cross-sectional study in Malawi. Trop Med Int Health TM IH. 2015;20:1549-58.

8. Vt H, Manigandan T, Sarumathi T, Aarthi Nisha V, Amudhan A. Dental considerations in pregnancy-a critical review on the oral care. J Clin Diagn Res JCDR. 2013;7:948-53.

9. Oliveira B, Nadanovsky P. The impact of oral pain on quality of life during pregnancy in low-income Brazilian women. J Orofac Pain. 2006;20:297-305.

10. Krüger M, Lang C, Almeida L, Correa F, Romano A, Pappen F. Dental pain and associated factors among pregnant women: an observational study. Matern Child Health J. 2014;19:504-10.

11. Shah AF, Batra M, Qureshi A. Evaluation of impact of pregnancy on oral health status and oral health related quality of life among women of Kashmir Valley. J Clin Diagn Res JCDR. 2017;11:ZC01-4.
12. Mjör IA, Odont D. Pulp-dentin biology in restorative dentistry.s Part 2: initial reactions to preparation of teeth for restorative procedures. Quintessence Int Berl Ger. 1985;2001(32):537-51.

13. Frencken JE, Pilot T, Songpaisan Y, Phantumvanit P. Atraumatic restorative treatment (ART): rationale, technique, and development. J Public Health Dent. 1996;56:135-40; discussion 161-163.

14. Frencken JE, Leal SC, Navarro MF. Twenty-five-year atraumatic restorative treatment (ART) approach: a comprehensive overview. Clin Oral Investig. 2012:16:1337-46.

15. Carvalho T-S, Ribeiro T-R, Bönecker M, Pinheiro E-C-M, Colares V. The atraumatic restorative treatment approach: an "atraumatic" alternative. Med Oral Patol Oral Cirugia Bucal. 2009;14:e668-73.

16. Shenoy R, Jain A, Mala K. Atraumatic restorative treatment for dental caries among pregnant women attending primary health centres: A small scale demonstration. J Interdiscip Dent. 2014;4:71-5.

17. Hamama H, Yiu C, Burrow M. Current update of chemomechanical caries removal methods. Aust Dent J. 2014;59:446-56; quiz 525.

18. Mobeen N, Jehan I, Banday N, Moore J, McClure EM, Pasha O, et al. Periodontal disease and adverse birth outcomes: a study from Pakistan. Am J Obstet Gynecol. 2008:198:514.e1-514.e8.

19. Wandera M, Åstrøm AN, Okullo I, Tumwine JK. Determinants of periodontal health in pregnant women and association with infants'anthropometric status: a prospective cohort study from Eastern Uganda. BMC Pregnancy Childbirth. 2012;12:90.

20. Opeodu OI, Dosumu EB, Arowojolu MO. Periodontal Condition and Treatment Needs of Some Pregnant Women in Ibadan. Nigeria Ann Med Health Sci Res. 2015;5:213-7.

21. Kashetty M, Kumbhar S, Patil S, Patil P. Oral hygiene status, gingival status, periodontal status, and treatment needs among pregnant and nonpregnant women: a comparative study. J Indian Soc Periodontol. 2018;22:164-70.

22. Jawa D, Singh S, Somani R, Jaidka S, Sirkar K, Jaidka R. Comparative evaluation of the efficacy of chemomechanical caries removal agent (Papacarie) and conventional method of caries removal: an in vitro study. J Indian Soc Pedod Prev Dent. 2010;28:73-7.

23. Singh S, Singh D, Jaidka S. Comparative clinical evaluation of chemomechanical caries removal agent Papacarie with conventional method among rural population in India - in vivo study. Braz J Oral Sci. 2011;10:193-8.

24. Maru VP, Shakuntala BS, Nagarathna C. Caries removal by chemomechanical (CarisolvTM) vs. rotary drill: a systematic review. Open Dent J. 2015:9:462-72

25. Almaz ME, Sönmez IŞ, Oba AA. Comparison of chemomechanical caries removal using Papacárie versus conventional method in children. Eur J Gen Dent. 2016;5:1.

26. Barata TJE, Bresciani E, Mattos MCR, Lauris JRP, Ericson D, Navarro MF de L. Comparison of two minimally invasive methods on the longevity of glass ionomer cement restorations: short-term results of a pilot study. J Appl Oral Sci Rev FOB. 2008;16:155-60.

27. Schulz KF, Altman DG, Moher D, for the CONSORT Group. CONSORT. Statement: updated guidelines for reporting parallel group randomised trials. Ann Int Med. 2010;2010:152.

28. Wewers ME, Lowe NK. A critical review of visual analogue scales in the measurement of clinical phenomena. Res Nurs Health. 1990;13:227-36.

29. Gugnani N, Pandit I, Srivastava N, Gupta M, Sharma M. International Caries Detection and Assessment System (ICDAS): a new concept. Int J Clin Pediatr Dent. 2011;4:93-100.

30. Loe H, Silness J. Periodontal disease in pregnancy. I. Prevalence and severity. Acta Odontol Scand. 1963;21:533-51.

31. Nadanovsky P, Cohen Carneiro F, Souz de Mello F. Removal of caries using only hand instruments: a comparison of mechanical and chemomechanical methods. Caries Res. 2001;35:384-9.

32. Faul F, Erdfelder E, Lang A-G, Buchner A. G*Power 3: a flexible statistical power analysis program for the social, behavioral, and biomedical sciences. Behav Res Methods. 2007;39:175-91.

33. Suresh K. An overview of randomization techniques: An unbiased assessment of outcome in clinical research. J Hum Reprod Sci. 2011;4:8-11.

34. Doig GS, Simpson F. Randomization and allocation concealment: a practical guide for researchers. J Crit Care. 2005;20:187-91; discussion 191-193.

35. Freitas MCC de A, Fagundes TC, Modena KC da S, Cardia GS, Navarro MF de L. Randomized clinical trial of encapsulated and hand-mixed 
glass-ionomer ART restorations: one-year follow-up. J Appl Oral Sci. 2018;26:e20170129. https://doi.org/10.1590/1678-7757-2017-0129.

36. Molina GF, Faulks D, Frencken J. Acceptability, feasibility and perceived satisfaction of the use of the Atraumatic Restorative Treatment approach for people with disability. Braz Oral Res. 2015;29(1):1-9. https://doi. org/10.1590/1807-3107BOR-2015.vol29.0097.

37. Abdul Khalek A, Elkateb MA, Abdel Aziz WE, El Tantawi M. Effect of papacarie and alternative restorative treatment on pain reaction during caries removal among children: a randomized controlled clinical trial. J Clin Pediatr Dent. 2017:41:219-24.

38. Khoshnevisan M, Albujeer A, Attaran N, Almahafdha A, Alaboudy A. WHO's oral health assessment questionnaire for adult: psychometric properties of the Arabic version. J Contemp Med Sci. 2016;2:116-18.

39. Petersen, Poul Erik, Baez, Ramon J \& World Health Organization. Oral health surveys: basic methods, 5 th ed. World Health Organization; 2013. https://apps.who.int/iris/handle/10665/97035.

40. Silness J, Loe H. Periodontal disease in pregnancy. II. Correlation between oral hygiene and periodontal condtion. Acta Odontol Scand. 1964;22:121-35.

41. Kumar J, Nayak M, Prasad KL, Gupta N. A comparative study of the clinical efficiency of chemomechanical caries removal using Carisolv ${ }^{\circledR}$ and Papacarie ${ }^{\circledR}$ —a papain gel. Indian J Dent Res. 2012;23:697.
42. Ericson D, Zimmerman M, Raber H, Götrick B, Bornstein R, Thorell J. Clinical evaluation of efficacy and safety of a new method for chemomechanical removal of caries. Caries Res. 1999;33:171-7.

43. Innes NPT, Frencken JE, Bjørndal L, Maltz M, Manton DJ, Ricketts D, et al. Managing carious lesions: consensus recommendations on terminology. Adv Dent Res. 2016;28:49-57.

44. Anegundi RT, Patil SB, Tegginmani V, Shetty SD. A comparative microbiological study to assess caries excavation by conventional rotary method and a chemo-mechanical method. Contemp Clin Dent. 2012;3:388-92.

45. Goyal PA, Kumari R, Kannan VP, Madhu S. Efficacy and tolerance of papain gel with conventional drilling method: a clinico-microbiological study. J Clin Pediatr Dent. 2015;39:109-12.

46. Bottega F, Bussadori S, Battisti I, Vieira E, Pompeo T, Winkelmann E. Costs and benefits of Papacarie in pediatric dentistry: a randomized clinical trial. Sci Rep. 2018;3:17908. https://doi.org/10.1038/s41598-018-36092-x.

\section{Publisher's Note}

Springer Nature remains neutral with regard to jurisdictional claims in published maps and institutional affiliations.
Ready to submit your research? Choose BMC and benefit from:

- fast, convenient online submission

- thorough peer review by experienced researchers in your field

- rapid publication on acceptance

- support for research data, including large and complex data types

- gold Open Access which fosters wider collaboration and increased citations

- maximum visibility for your research: over $100 \mathrm{M}$ website views per year

At BMC, research is always in progress.

Learn more biomedcentral.com/submissions 